\title{
Efeito do vínculo com um médico de família no controle da pressão arterial em hipertensos
}

\author{
Effect of family doctor relationship on blood pressure control in hypertensive patients \\ Efecto del vínculo con un médico de familia en el control de la presión arterial en hipertensos
}

\author{
Andre Klafke ${ }^{\mathrm{a}}$, Laura Afanador Pineros Vaghetti ${ }^{\mathrm{a}}$, Andre Dias Costa ${ }^{\mathrm{a}}$
}

\section{Resumo}

Objetivo: Avaliar a associação entre vínculo com um médico de família e controle da pressão arterial em hipertensos de duas unidades de saúde de Porto Alegre, RS. Métodos: Estudo transversal com uma amostra aleatória de 128 hipertensos de 18 anos ou mais de duas unidades de saúde, nos quais foi aplicado um questionário para avaliar suas características e a presença ou não de vínculo com um médico de família, e aferidos pressão arterial, peso e altura, entre março e setembro de 2016. Foi realizada regressão de Poisson para controle de possíveis fatores de confusão entre vínculo e controle da pressão. Resultados: A população estudada era acompanhada pelas unidades em média há 15 anos, era predominantemente idosa, do sexo feminino, branca, com sobrepeso, não tabagista, sedentária, aderente ao tratamento, $68,0 \%$ possuíam vínculo com um médico e $61,7 \%$ estavam com a pressão controlada. A presença de vínculo com um médico foi associada a um controle da pressão arterial $48 \%$ maior, controlado para possíveis fatores de confusão. Conclusão: O vínculo com um médico é uma ferramenta de baixo custo que permite melhorar o controle pressórico em pacientes hipertensos - controle este importante para a redução das complicações cardiovasculares.

\section{Abstract}

Objective: To evaluate the relation between a family doctor-patient relationship and blood pressure control in hypertensive patients from two health units in Porto Alegre, Rio Grande do Sul. Methods: A cross-sectional study with a random sample of 128 hypertensive 18 years of age and older patients from two health units, in which a questionnaire was applied to evaluate their characteristics and the presence or absence of a link with a family doctor, while blood pressure, weight and height were measured between March and September 2016. Poisson regression was performed to control possible confounding factors between family doctor-patient relationship and blood pressure control. Results: The population studied was monitored by the health units during 15 years on average and was predominantly elderly, female, white, overweight, non-smoker, sedentary, adherent to treatment, while $68.0 \%$ had a relationship with a family doctor and $61.7 \%$ had controlled blood pressure. The presence of a relationship with a doctor was associated with a blood pressure control $48 \%$ higher, controlled for possible confounding factors. Conclusion: Doctor-patient relationship is a low cost tool in medical practice that allows better blood pressure control in hypertensive patients - a control which is important in reducing cardiovascular complications.
Palavras-chave: Hipertensão/prevenção $\&$ controle Relações Médico-Paciente Adesão à Medicação

\section{Keywords:}

Hypertension/prevention \& control Physician-Patient Relations Medication Adherence
Como citar: Klafke A, Vaghetti LAP, Costa AD. Efeito do vínculo com um médico de família no controle da pressão arterial em hipertensos. Rev Bras Med Fam Comunidade. 2017;12(39):1-7. http://dx.doi.org/10.5712/rbmfc12(39)1444
Fonte de financiamento: declaram não haver. Parecer CEP: não se aplica. Conflito de interesses: declaram não haver. Procedência e revisão por pares: revisado por pares. Recebido em: 09/01/2017. Aprovado em: 04/10/2017. 


\section{Resumen}

Objetivo: Evaluar la asociación entre el vínculo con un médico de familia y el control de la presión arterial en hipertensos de dos unidades de salud de Porto Alegre, Rio Grande do Sul. Métodos: Estudio transversal con una muestra aleatoria de 128 hipertensos mayores de 18 años de dos unidades de salud, en los cuales fue aplicado un cuestionario para evaluar sus características y la presencia o no de un vínculo con un médico de familia y la medida de la presión arterial, peso y altura entre marzo y septiembre de 2016. Fue realizada una regresión de Poisson para el control de los posibles factores de confusión entre el vínculo y el control de la presión. Resultados: La población estudiada era acompañada por las unidades en una media de 15 años, predominantemente de edad avanzada, de sexo femenino, blanca, con sobrepeso, no fumadora, sedentaria, con adhesión al tratamiento, y el $68,0 \%$ tenía vínculo con un médico y $61,7 \%$ estaba con la presión controlada. La presencia del vínculo con un médico fue asociada a un control de la presión arterial $48 \%$ mayor, controlada para posibles factores de confusión. Conclusión: El vínculo con un médico de familia es una herramienta de bajo costo, que permite mejorar el control tensional en pacientes hipertensos, el cual es importante para la reducción de las complicaciones cardiovasculares.

\section{Palabras clave:}

Hipertensión/prevención

\& control

Relaciones Médico-Paciente

Cumplimiento de la Medicación

\section{Introdução}

A hipertensão arterial sistêmica (HAS) tem alta prevalência na população adulta e consiste em um dos principais fatores de risco cardiovascular. ${ }^{1}$ Entretanto, seu controle, geralmente realizado a partir do acompanhamento em unidades de saúde, pode reduzir suas complicações. ${ }^{2,3}$

Na Atenção Primária à Saúde (APS), o vínculo é uma percepção de "ligação" que surge na relação que se estabelece entre o médico e seu paciente. Essa ligação é importante tanto para o paciente ter a percepção de cuidado quanto para o médico fortalecer a percepção de responsabilidade e compromisso com o cuidado do seu paciente. ${ }^{4}$

Apesar de este tema, "vínculo", permear a APS desde seus primórdios, não encontramos muitos estudos sobre ele na literatura - talvez por nem haver um termo descritor para ele, com este sentido, indexado na literatura médica. Aparecem mais estudos sobre esse assunto desde 2004, aplicados em publicações de Saúde Coletiva ligadas a doenças transmissíveis como AIDS e tuberculose, saúde mental, saúde da mulher e da criança e, em menor proporção, a doenças crônicas como HAS e diabetes melito (6\% das publicações). ${ }^{5} \mathrm{~A}$ hipótese seria de que o vínculo poderia melhorar o controle de doenças crônicas. Entretanto, talvez até pela inexistência de termo indexado referente a ele na literatura, encontramos poucos estudos avaliando esta relação.

Existem várias maneiras de organizar a APS na rede pública de um país. No Brasil, desde 1994, com o início do Programa de Saúde da Família, optou-se pela adscrição dos pacientes a uma unidade de saúde, conforme a região de moradia, criando, assim, um vínculo do indivíduo com esta Unidade. Entretanto, as unidades de saúde podem comportar mais de uma equipe, com mais de um médico, portanto. ${ }^{6} \mathrm{Em}$ algumas delas, o paciente pode acabar consultando cada vez com um médico diferente.

O vínculo é uma tecnologia leve e barata. ${ }^{7}$ Se estiver efetivamente associado ao controle pressórico, seria valioso investir nele como uma ferramenta para o controle da pressão arterial (PA) em hipertensos de unidades de saúde com mais de um médico. Diante disso, o objetivo deste estudo é avaliar se a percepção de vinculação com um médico de família e comunidade está associada a um melhor controle da PA em hipertensos de duas unidades de saúde de Porto Alegre, assim como identificar outros fatores que possam estar associados ao controle desta doença. 


\section{Métodos}

Foi realizado um estudo transversal com a população adscrita de duas unidades de saúde de Porto Alegre, RS, que abrangem 23.216 pessoas. Os usuários das Unidades são predominantemente de classe média, com significativa proporção de idosos. As unidades de saúde têm mais de 25 anos de existência, são unidades-escola, com alguns médicos que atuam desde o início de sua implantação e outros há menos tempo, além de médicos residentes e estagiários de medicina, que atendem sob supervisão dos preceptores.

Os critérios de inclusão foram morar na área de abrangência de (e estar cadastrado em) uma das unidades de saúde, ter idade de 18 anos ou mais, estar cadastrado na ação programática do HiperDia com diagnóstico de HAS havia pelo menos um ano e fazer acompanhamento para HAS nas unidades. Para ser cadastrado no HiperDia, o paciente devia ter a média das pressões arteriais adequadamente aferidas ${ }^{3}$ acima de 140/90 mmHg, confirmada em pelo menos mais um encontro, ou ter vindo de outro serviço com diagnóstico de HAS e em uso de anti-hipertensivos. Preencheram os critérios de inclusão 3.197 pessoas.

De acordo com a lista dos usuários cadastrados no Programa HiperDia, foi realizada uma amostragem aleatória simples. O tamanho de amostra foi calculado conforme Hulley, utilizando uma magnitude padronizada de efeito de 0,5, alfa bidirecional de 0,05 e beta de 0,20. ${ }^{8}$ A partir deste cálculo, foi selecionada com o programa SPSS versão 19.0 uma amostra de 128 hipertensos, 98 de uma unidade e 30 de outra (uma unidade tinha a população maior que a outra).

Foi aplicado aos pacientes um questionário estruturado avaliando as variáveis sexo, idade, raça (autorreferida), ingestão de bebida alcoólica (tipo de bebida e quantidade semanal), tabagismo (número de cigarros consumidos por dia atualmente), prática de atividade física (tempo de atividade física semanal) e um questionário específico sobre adesão ao tratamento para hipertensão. ${ }^{9}$ Este questionário apresenta quatro dimensões relacionadas à adesão, com 24 itens, numa escala Likert de 5 pontos, que permite classificar os pacientes em 3 categorias, conforme seu nível de adesão: "não adesão total" (24 a 48 pontos), "risco para não adesão" (49 a 72 pontos) e "adesão" (73 a 120 pontos).

O peso foi aferido em balanças mecânicas, e a altura, em antropômetro vertical. O índice de massa corporal (IMC) foi calculado como a razão entre o peso (em quilogramas) e o quadrado da altura (em metros). O valor da variável PA considerada foi a média entre três aferições: as duas últimas registradas no prontuário e a verificada pelos examinadores. A PA foi aferida pelos examinadores conforme preconizado pela $7^{\text {a }}$ Diretriz Brasileira de Hipertensão Arterial.

A coleta dos dados foi realizada por dois dos autores, na unidade de saúde e no domicílio dos pacientes, sendo realizado um treinamento para sua calibração para a coleta dos dados. Os dados coletados foram codificados, digitados em planilhas Excel versão 2013 e exportados para o programa SPSS 19.0, no qual foram analisados.

Foi realizada inicialmente análise univariada, depois bivariada entre as variáveis independentes e a dependente (controle da PA, dicotomizada como controlada, se $<140 / 90 \mathrm{mmHg}$, ou não controlada). $\mathrm{Na}$ análise bivariada, os testes t e qui-quadrado foram utilizados para avaliar a força e significância estatística das associações. As variáveis com $p<0,20$ na análise bivariada foram incluídas na análise multivariada do tipo regressão de Poisson. Foi avaliada também presença de multicolinearidade entre as variáveis a partir da estatística do fator de inflação de variância (VIF). 
O Projeto foi aprovado pelo Comitê de Ética em Pesquisa do Grupo Hospitalar Conceição. O Termo de Consentimento Livre e Esclarecido (TCLE) foi apresentado em duas vias, sendo uma delas entregue ao sujeito de pesquisa ou responsável após a assinatura.

\section{Resultados}

Dentre os 128 pacientes sorteados para participarem do estudo, 11 não preenchiam o critério de inclusão: 2 haviam falecido, 1 não era hipertenso (fora erroneamente cadastrado por alguém da equipe no programa HiperDia como hipertenso) e 8 não faziam acompanhamento na unidade. Adicionalmente, houve seis perdas reais do sorteio - quatro pacientes que não puderam ser localizados e dois que se recusaram a participar - representando 4,7\% da amostra. Esses 17 pacientes foram substituídos em outro sorteio.

Os 128 pacientes selecionados foram entrevistados e examinados no período de março a setembro de 2016. A população estudada tinha idade mais avançada, era predominantemente do sexo feminino, branca e a maioria apresentava sobrepeso (Tabela 1). Poucos eram tabagistas, pouco mais de um terço costumava beber ao menos semanalmente e a maioria era sedentária e aderente ao tratamento: nenhum indivíduo foi classificado em "não adesão total" (24 a 48 pontos), em nenhum grupo; apenas 3 (2,3\%) com PA não controlada e nenhum com PA controlada tinham "risco para não adesão" (49 a 72 pontos); e os demais foram considerados "aderentes" (73 a 120 pontos). A maior parte fazia acompanhamento na mesma unidade havia mais de 10 anos, apresentava vínculo com um dos médicos da unidade, e 79 $(61,7 \%)$ estavam com a pressão controlada na média das três últimas aferições.

Tabela 1. Características dos pacientes com hipertensão arterial sistêmica de duas unidades de saúde de Porto Alegre, 2016 *

\begin{tabular}{|c|c|c|c|c|}
\hline & PA controlada & PA não controlada & Total & Valor-p ${ }^{\ddagger}$ \\
\hline Idade (anos) & $67,8 \pm 12,6$ & $68,6 \pm 12,3$ & $68,1 \pm 12,4$ & 0,721 \\
\hline Sexo & & & & 0,345 \\
\hline masculino & $17(21,5 \%)$ & $15(30,6 \%)$ & $32(25 \%)$ & \\
\hline feminino & $62(78,5 \%)$ & $34(69,4 \%)$ & $96(75 \%)$ & \\
\hline Cor & & & & 1,000 \\
\hline branca & $69(87,3 \%)$ & $42(85,7 \%)$ & $111(86,7 \%)$ & \\
\hline não branca & $10(12,7 \%)$ & $7(14,3 \%)$ & $17(13,3 \%)$ & \\
\hline $\mathrm{IMC}^{\dagger}$ & & & & 0,887 \\
\hline magreza & $1(1,3 \%)$ & $1(2,0 \%)$ & $2(1,6 \%)$ & \\
\hline eutrofia & $15(19,0 \%)$ & $13(26,5 \%)$ & $28(21,9 \%)$ & \\
\hline sobrepeso & $47(59,5 \%)$ & $20(40,8 \%)$ & $67(52,3 \%)$ & \\
\hline obesidade $(\geq 30)$ & $16(20,3 \%)$ & $15(30,6 \%)$ & $31(24,2 \%)$ & \\
\hline Tabagismo & $7(8,9 \%)$ & $5(10,2 \%)$ & $12(9,4 \%)$ & 1,000 \\
\hline Consumo de bebida alcoólica & $23(29,1 \%)$ & $22(44,9 \%)$ & $45(35,2 \%)$ & 0,104 \\
\hline Prática de atividade física & $20(25,3 \%)$ & $15(30,6 \%)$ & $35(27,3 \%)$ & 0,653 \\
\hline Escore de adesão ao tratamento & $95,5 \pm 8,6$ & $91,3 \pm 12,5$ & $93,9 \pm 10,4$ & 0,046 \\
\hline Tempo de acompanhamento na unidade (anos) & $16,6 \pm 8,7$ & $14,2 \pm 9,2$ & $15,7 \pm 8,9$ & 0,133 \\
\hline Vínculo com um médico na unidade & $61(77,2 \%)$ & $26(53,1 \%)$ & $87(68,0 \%)$ & 0,008 \\
\hline Total & $79(61,7 \%)$ & $49(38,3 \%)$ & $128(100 \%)$ & \\
\hline
\end{tabular}

PA controlada: pressão arterial < 140/90 mmHg; PA não controlada: pressão arterial $\geq 140 / 90 \mathrm{mmHg}$ * valores em média \pm desvio padrão ou n (\%); †índice de massa corporal, em kg/m². Magreza: $<18,5$; eutrofia: 18,5-24,9; sobrepeso: $25-29,9$; obesidade: $\geq 30 .{ }^{\ddagger}$ da análise bivariada, com controle da pressão arterial. Teste t para igualdade de médias para amostras independentes para as variáveis contínuas e teste qui-quadrado de Pearson com correção de continuidade para variáveis categóricas. 
Na análise bivariada, apresentaram associação com o controle da PA, com um nível de significância inferior ao predeterminado para entrar na análise multivariada $(p<0,20)$, as variáveis presença de vínculo com um médico na unidade $(p=0,008)$, escore de adesão ao tratamento $(p=0,046)$ e consumo de bebida alcoólica $(p=0,104)$. Antes da estimativa de parâmetros na regressão de Poisson, foi realizada análise de colinearidade entre todas as variáveis, não se verificando colinearidade entre nenhuma delas.

A regressão de Poisson mostrou que, dentre todas as variáveis incluídas na análise, apenas uma apresentou associação estatisticamente significativa com o controle da PA. A presença de vínculo com um médico na unidade foi associada a uma proporção $48 \%$ maior de controle da PA em relação aos pacientes sem vínculo (RR 1,482, $p=0,04$ ), controlada para os possíveis fatores de confusão.

\section{Discussão}

A percepção de existência de vínculo com um médico específico, na unidade de saúde, foi o único fator associado a maior controle da PA neste estudo. Não foram encontrados outros trabalhos avaliando o efeito do vínculo com um médico no controle pressórico de pacientes hipertensos. Apesar de estudos sobre esse assunto terem sido buscados com diferentes termos na revisão da literatura, não há um descritor indexado para "vínculo" no sentido que utilizamos, o que pode ter impedido a localização de eventuais trabalhos sobre isso.

Entretanto, outros estudos avaliaram os efeitos positivos do vínculo. Um estudo em Rio Grande, no Sul do Brasil, concluiu que a existência de um médico de referência está associada a maior probabilidade de realização de exames preventivos em homens e mulheres. ${ }^{10}$ Outro estudo, que avaliou relação entre confiança no médico e adoção de estilos de vida e adesão a medicamentos para tratamento da HAS, encontrou associação positiva para redução de peso, mas não para redução na ingestão de sal, aumento na atividade física ou adesão ao tratamento medicamentoso. ${ }^{11}$

Vários fatores podem explicar o potencial do vínculo em aumentar o controle da PA em pacientes hipertensos. O vínculo presume uma relação de compreensão, apoio, escuta e estímulo à narração. ${ }^{12}$ Sendo assim, estimula uma relação horizontal, de confiança, com liberdade para o paciente tirar suas dúvidas. O vínculo também permite ao médico conhecer seu paciente integralmente, inclusive em seu contexto familiar e social, possibilitando-lhe identificar dificuldades deste e, com isso, estimulá-lo a ser protagonista de seu tratamento. A relação de confiança surge quando "qualificamos a natureza" dos afetos de duas pessoas que "descobrem que mutuamente se convêm". São estes os "afetos que dão consistência ao vínculo" e aos laços sociais. ${ }^{13}$

Um estudo sobre vínculo e tratamento da tuberculose concluiu que o vínculo entre paciente e profissionais da saúde favorece a adesão ao tratamento desta doença. ${ }^{14}$ Outro trabalho concluiu que a aliança terapêutica (dimensões cognitiva e emocional da relação médico-paciente) está fortemente associada à adesão e ao nível de satisfação do paciente com o tratamento. ${ }^{15}$

Nesse sentido, o melhor controle pressórico em pacientes com vínculo com um médico poderia ser explicado não apenas pelo vínculo em si, mas por uma maior adesão ao tratamento nestes pacientes. Entretanto, ao contrário dos estudos que obtiveram essa relação, mas em conformidade com outro, ${ }^{11}$ não encontramos associação entre vínculo e adesão. Isso pode ter ocorrido porque quase todos os pacientes 
encontravam-se na categoria de "aderentes" ao tratamento. Contudo, como sua pontuação variava, dentro desta categoria, pode também indicar que o vínculo pode melhorar o controle pressórico independentemente de sua influência na adesão ao tratamento - o que precisaria ser melhor avaliado em outro estudo.

Cabe ressaltar a importância da comunicação no tratamento de doenças crônicas como a HAS, para manter uma relação efetiva com os pacientes. Um estudo que avaliou a opinião de hipertensos encontrou alto grau de ignorância destes quanto à doença e concluiu que, se os médicos tivessem mais tempo para os pacientes, poderiam receitar menos medicamentos. ${ }^{15}$ Outro estudo concluiu que a restrição da comunicação a aspectos biomédicos contribui para uma relação impessoal, sem diálogos, escutas, responsabilização e subjetividades, dificultando assim o estabelecimento do vínculo. ${ }^{14}$

Os pontos fortes deste estudo foram sua realização em unidades de saúde com longa trajetória e experiência, médicos trabalhando nelas há muitos e poucos anos, a presença de residentes em processo de formação, a seleção aleatória de participantes e perdas de apenas $4,7 \%$, reduzindo assim a probabilidade de vieses de seleção. $O$ fato de o estudo ter sido realizado em apenas duas unidades de saúde de uma mesma cidade e de estas serem unidades-escola pode fazer com que os resultados não possam ser extrapolados para toda a população brasileira.

A utilização de medidas de PA do prontuário para calcular a PA média dos indivíduos também pode constituir uma limitação, embora todos os médicos do Serviço tivessem recebido capacitação recente sobre aferição da PA devido a outro estudo realizado havia pouco na Instituição. Apesar de ter sido utilizado um instrumento validado para a aferição da adesão, sempre há a possibilidade de um viés de informação.

A contribuição deste estudo consiste em iniciar na literatura a avaliação e documentação da importância do vínculo com um mesmo médico no controle da pressão arterial em hipertensos. Esta associação passa a ser mais relevante com o fortalecimento da APS, na medida em que mais hipertensos são acompanhados neste nível do sistema e mais unidades de saúde com mais de um médico de família são estabelecidas. Entretanto, mais estudos são necessários para avaliar se essa associação ocorre também em outros locais do País.

\section{Conclusão}

O vínculo é o resultado de um processo de interação entre o profissional de saúde e o usuário, que vai se consolidando e compartilhando a responsabilidade de ambos no processo saúde-doença. Neste processo, o vínculo é uma ferramenta que fortalece as trocas de saberes, o conhecimento científico com o popular, que, somados, integram a relação médico-paciente e comprometem ambas partes a assumirem os cuidados de saúde. Os resultados deste estudo reafirmam a importância da relação interpessoal e seu impacto positivo em várias doenças, sendo o vínculo importante para a qualificação da prática profissional numa perspectiva mais ampliada e no controle de doenças crônicas, como a HAS.

Por o vínculo ser uma tecnologia leve bastante barata, seu uso deve ser estimulado para melhorar o controle pressórico em pacientes hipertensos. A melhora do controle pressórico está associada a uma redução das complicações cardiovasculares, efeito muito relevante tanto em nível individual quanto coletivo, inclusive em custos ao sistema de saúde..$^{1,12}$ 


\section{Referências}

1. Iser BPM, Moura L, Schmidt MI, Duncan BB. Estratégias preventivas para as doenças crônicas não transmissíveis. In: Duncan BB, Schmidt MI, Giugliani ERJ, Duncan MS, Giugliani C. Medicina ambulatorial: Condutas de Atenção Primária Baseadas em Evidências. 4ª ed. Porto Alegre: Artmed; 2013. p. 530-5.

2. Car MR, Pierin AMG, Aquino VLA. Estudos sobre a influência do processo educativo no controle da hipertensão arterial. Rev Esc Enferm USP. 1991;25(3):259-69. DOI: http://dx.doi.org/10.1590/0080-6234199102500300259

3. Sociedade Brasileira de Cardiologia/Sociedade Brasileira de Hipertensão/Sociedade Brasileira de Nefrologia. VI Diretrizes Brasileiras de Hipertensão. Arq Bras Cardiol. 2010;95(1 Supl.1):1-51.

4. Rocha BV, Gazim CC, Pasetto CV, Somões CJ. Relação Médico-Paciente. Rev Med Res. 2011;13(2):114-8.

5. Brunello MEF, Ponce MAZ, Assis EG, Andrade RLP, Scatena LM, Palha PF, et al. O Vínculo na atenção à saúde: revisão sistematizada na literatura, Brasil (1998-2007). Acta Paul Enferm. 2010;23(1):131-5. DOI: http://dx.doi.org/10.1590/S0103-21002010000100021

6. Brasil. Brasil Ministério da Saúde. Portaria № 2.436, de 21 de setembro de 2017. Aprova a Política Nacional de Atenção Básica, estabelecendo a revisão de diretrizes para a organização da Atenção Básica, no âmbito do Sistema Único de Saúde (SUS). Brasília: Diário Oficial da União; 2017.

7. Cunha EM. Vínculo Longitudinal na Atenção Primária: avaliando os modelos assistenciais do SUS [Tese de doutorado]. Rio de Janeiro: Fundação Oswaldo Cruz; 2009.

8. Browner WS, Newman TB, Cummings SR, Hulley SB. Estimando o Tamanho de Amostra e o Poder Estatístico: Pontos Básicos. In: Hulley SB, Cummings SR, Browner WS, Grady D, Hearst N, Newman TB. Delineando a Pesquisa Clínica. 2ª ed. Porto Alegre: Artmed; 2003. p. 83-110.

9. Borges JWP. Instrumento de Avaliação da Não Adesão ao Tratamento da Hipertensão arterial: desenvolvimento e validação de conteúdo [Dissertação de mestrado]. Fortaleza: Universidade Estadual do Ceará; 2012.

10. Mendoza-Sassi R, Béria JU. Prevalência do médico de referência, fatores associados e seu efeito na utilização de serviços de saúde: um estudo de base populacional no Sul do Brasil. Cad Saúde Pública. 2003;19(5):1257-66. DOI: http://dx.doi.org/10.1590/S0102$311 \times 2003000500004$

11. Jones DE, Carson KA, Bleich SN, Cooper LA. Patient trust in physicians and adoption of lifestyle behaviors to control high blood pressure. Patient Educ Couns. 2012;89(1):57-62. DOI: http://dx.doi.org/10.1016/j.pec.2012.06.003

12. Fuertes JN, Mislowack A, Bennett J, Paul L, Gilbert TC, Fontan G, et al. The physician-patient working alliance. Patient Educ Couns. 2007;66(1):29-36. DOI: http://dx.doi.org/10.1016/j.pec.2006.09.013

13. Teixeira RR. Humanização e Atenção Primária à Saúde. Ciênc Saúde Coletiva.2005;10(3):585-97.DOI: http://dx.doi.org/10.1590/S141381232005000300016

14. Brunello MEF, Cerqueira DF, Pinto IC, Arcênio RA, Gonzales RIC, Villa TCS, et al. Vínculo doente-profissional de saúde na atenção a pacientes com tuberculose. Acta Paul Enferm. 2009;22(2):176-82. DOI: http://dx.doi.org/10.1590/S0103-21002009000200010

15. Buendía JA. Actitudes, conocimientos y creencias del paciente hipertenso sobre la medicación antihipertensiva. Biomédica. 2012;32(4):578-84.

a Programa de Residência Médica de Medicina de Família e Comunidade, Grupo Hospitalar Conceição. Porto Alegre, RS, Brasil. andreklafke@ hotmail.com; lauraafa@gmail.com (Autora correspondente); diascostaandre@gmail.com 